\title{
La resurgence du populisme en Bolivie "Conscience de la Patrie" ou la construction de nouvelles identités urbaines dans un contexte compétitif
}

El resurgimiento del populismo en Bolivia

"Conciencia de patria" o la construcción de nuevas identidades urbanas en un contexto competitivo

The Resurgence of Populism in Bolivia

"Conscience of the Fatherland" Or the Construction of New Urban Identities in a Competitive Context

\section{Stéphanie Alenda}

\section{OpenEdition \\ Journals}

Edición electrónica

URL: http://journals.openedition.org/bifea/7228

DOI: $10.4000 /$ bifea.7228

ISSN: 2076-5827

Editor

Institut Français d'Études Andines

Edición impresa

Fecha de publicación: 1 abril 2001

Paginación: 1-26

ISSN: 0303-7495

Referencia electrónica

Stéphanie Alenda, «La resurgence du populisme en Bolivie

"Conscience de la Patrie" ou la construction de nouvelles identités urbaines dans un contexte compétitif », Bulletin de l'Institut français d'études andines [En línea], 30 (1) | 2001, Publicado el 08 abril 2000, consultado el 08 diciembre 2020. URL : http://journals.openedition.org/bifea/7228; DOI :

https://doi.org/10.4000/bifea.7228

\section{(c) (i) $\odot$}

Les contenus du Bulletin de l'Institut français d'études andines sont mis à disposition selon les termes de la licence Creative Commons Attribution - Pas d'Utilisation Commerciale - Pas de Modification 4.0 International. 


\title{
LA RESURGENCE DU POPULISME EN BOLIVIE “Conscience de la Patrie" ou la construction de NOUVELLES IDENTITÉS URBAINES DANS UN CONTEXTE COMPÉTITIF
}

Stéphanie ALENDA*

\begin{abstract}
Résumé
À travers l'étude du cas de "Conscience de la Patrie" (CONDEPA), parti politique bolivien fondé en 1988 à la suite de la fermeture des médias de Carlos Palenque, il s'agit d'examiner la pertinence pour la compréhension des mouvements dits "néo-populistes" de deux postulats des théories du populisme latino-américain : l'absence de conscience de classe des couches populaires et leur attachement à des valeurs traditionnelles, dites "prémodernes". L'étude du condépisme ne valide pas l'hypothèse d'une mobilisation populaire synonyme de résistance au changement. Elle ne valide pas non plus l'inverse, à savoir l'uniformisation des masses apathiques sous l'effet des médias. S'il apparaît que ce sont les plus discriminés qui ont les plus fortes chances de voter pour CONDEPA, on observe également que, dans cette catégorie, les plus prédisposés à soutenir le parti sont aussi les mieux intégrés à l'univers urbain où ils mettent en œuvre des tactiques individuelles d'ascension sociale. L" "autonomie " des acteurs condépistes, bien qu'ils appartiennent à un mouvement au verticalisme marqué, rend en effet quelque peu réductrice la perception du social en termes de reproduction des inégalités. L'étude du lien entre le leader et ses bases met au contraire en évidence que des mécanismes d'identification (à " Radio Télévision Populaire ", CONDEPA ou encore Carlos Palenque) contribuent à organiser une réalité sociale incertaine dans un contexte compétitif d'érosion des identités collectives.
\end{abstract}

Mots clés : Populisme, Bolivie, CONDEPA, identification partisane, El Alto, Cholas, sociologie politique, constructions identitaires.

\section{EL RESURGIMIENTO DEL POPULISMO EN BOLIVIA \\ “Conciencia de Patria” o la construcción de nuevas identidades urbanas EN UN CONTEXTO COMPETITIVO}

\section{Resumen}

A través del estudio de "Conciencia de Patria" (CONDEPA), partido político boliviano fundado en 1988 tras la clausura de los medios de comunicación de su leader Carlos Palenque, se trata de examinar la pertinencia de dos postulados de las teorías del populismo latinoamericanolos

${ }^{*}$ Université de Lille 1. Centre Lillois d'Études et de Recherches Sociologiques et Économiques (CLERSE). E-mail : stephanie.alenda@wanadoo.fr 
para comprender los movimientos denominados neopopulistas: la carencia de conciencia de clase atribuida a las capas populares y su apego a valores tradicionales o premodernos. El estudio del condepismo no valida la hipótesis de una movilización popular sinónima de resistencia al cambio. Tampoco valida lo contrario, es decir la uniformización de las masas apáticas bajo el efecto de los medios de comunicación. Si bien los que más tienden a votar por CONDEPA son los más discriminados, en esta categoría, los más propensos a apoyar al partido son también los mejor integrados en el universo urbano donde desarrollan tácticas individuales de ascenso. En efecto, la "autonomía" de los actores condepistas, a pesar de pertenecer a un movimiento vertical, vuelve algo reductora la percepción de lo social en términos de reproducción de las desigualdades. Al contrario, el estudio del vínculo entre el líder y sus bases hace resaltar unos mecanismos de identificación (con "Radio Televisión Popular", CONDEPA o Carlos Palenque) que contribuyen a organizar una realidad social incierta en un contexto competitivo de erosión de las identidades colectivas.

Palabras claves: Populismo, Bolivia, CONDEPA, identificación partidaria, El Alto, cholas, sociología política, construcciones identitarias.

\title{
THE RESURGENCE OF POPULISM IN BOLIVIA \\ “Conscience of the FatherLand" OR the Construction of New Urban Identities in a Competitive ConteXt
}

\begin{abstract}
This study examines the relevance of two assumptions held by theories of LatinAmerican populism to the study of the so called neo-populist movements. One, being a popular attachment to traditional values, presented by some as premodern, and the other, being a lack of popular class conscience. This will be approached addressing the electoral mobilisation of the Conscience of the Fatherland (CONDEPA), a Bolivian political party founded in 1988 following the closure of the media belonging to its leader Carlos Palenque. This study of Condepism does not validate the hypothesis that popular mobilisation is synonymous with a resistance to change. Neither does it validate the opposite, that is to say, the uniformization of apathetic masses subject to the influence of mass media. Rather, it intends to demonstrate that even though those who are the most discriminated against are also the most likely to vote for CONDEPA. Within this category, those who are better integrated into the urban universe where they adopt individual strategies towards social progress, are more inclined to support the party. The "autonomy" of these Condepists, despite their allegiance to a hierarchical party, belies the narrow perception held by some, that the social reality does nothing but perpetuate inequality. On the contrary, an analysis of the relationship between the leader and his supporters reveals that their identification with "Radio Televisión Popular", CONDEPA or Carlos Palenque enables them to forge a sense of order out of an uncertain social reality. All this is situated in a competitive context of erosion of collective identities.
\end{abstract}

Keywords: Populism, Bolivia, CONDEPA, Party Identification, El Alto, cholas, Political sociology, Construction of Identities.

\section{INTRODUCTION}

En Amérique Latine, le retour à la démocratie dans les années quatre-vingt s'accompagne d'une intensification de la crise sociale, elle-même aggravée par la crise financière et économique. L'État au bord de la banqueroute abjure le populisme, 
renonçant à son modèle d'industrialisation substitutive d'importations, et se soumet aux lois du marché sous la férule du Fond Monétaire International. S'ensuit une politique d'ajustement structurel dont le bilan social se révèle désastreux bien qu'elle obtienne des résultats macro-économiques spectaculaires.

Le cas de la Bolivie est particulièrement représentatif de ce processus. En effet, le retour à un régime démocratique en 1982 va de pair avec la volonté de rationaliser la bureaucratie et de réorganiser l'économie. La " Nouvelle Politique Économique" néolibérale contribue à redéfinir les contours de la nation bolivienne présentée à cette date par la Confédération des chefs d'entreprise privée (CEPB) comme " une conjonction de la démocratie représentative et pluraliste avec une modernisation de type néolibéral " (Mansilla, 1995 : 165). Cependant, même si ces mesures tendent à relancer une croissance soutenue mais qui demeure néanmoins modérée et assez fragile (moins de $1 \%$ en 1999) et réussissent à réduire l'hyperinflation qui passe de $23500 \%$ en 1985 à $3,1 \%$ en 1999 (Bonnet, 19. 09. 2000), leur coût social demeure très élevé : entre 1985 et 1989, le décret 21060 pris par le gouvernement de Victor Paz Estenssoro (MNR) multiplie par deux le taux de sous-emploi et conduit à une croissance effrénée du secteur informel (Mesa, 1992 : 78-80).

Dans ce contexte, la symbolique nationale de 1952 devient obsolète. L'Étatnation intégrationniste et clientéliste - pratiques clientélaires que la classe politique rend responsables de la dictature - qu'avait forgé la Révolution est remis en question par les partis politiques (Mouvement Nationaliste Révolutionnaire et Action Démocratique Nationaliste) qui souscrivent le " Pacte pour la Démocratie " en octobre 1985. Il est d'ailleurs emblématique que ce soient le même homme et le même parti qui avaient édifié l'État populiste en 1952 qui procèdent à son démantèlement en 1985 (Favre, 1996 : 120-121), dans un contexte où le modèle de développement épuisé des années 30-50 laisse place à une politique de déconcentration et de dénationalisation.

Cependant, et paradoxalement, cette modernisation de l'État voit réapparaître dans différents pays latino-américains des mouvements qualifiés de " néo-populistes ", préfixe qui prétend essentiellement marquer une différence entre les nouveaux États néolibéraux (et néo-populistes) et les politiques nationalistes étatistes caractéristiques du populisme (Taguieff, 1996). En réadaptant à cette nouvelle conjoncture un postulat propre à la théorie structurelle-fonctionnaliste (1), on pourrait émettre l'hypothèse que les néo-populismes — au même titre que les populismes — remettent en question le processus de modernisation de l'État en démontrant la permanence de structures archaïques (que Germani qualifie de " traditionnelles " et Di Tella de " féodales ") qui agiraient comme un frein à la modernisation. C'est notamment ce que soutient l' historien espagnol Álvarez Junco lorsqu'il présente ce type de mobilisation comme une contre-réaction aux politiques de modernisation qui impliqueraient la désagrégation des solidarités traditionnelles :

“(...) en s'adressant aux partisans de ces mouvements comme au

' peuple' et en les appelant ' colonne vertébrale de la patrie ', le dirigeant

(1) Cf. Moscoso Perea, 1990. Cette dénomination renvoie aux ouvrages de Germani, 1962 ; 1978 et Di Tella et al., 1965. 
populiste leur offre un sens de communauté et un ensemble de croyances qui les protègent face au désarroi que produit la modernité, face à la liquidation de la vision religieuse du monde et des liens et formes de vie traditionnels." (1994: 27)

Cette lecture relativement récente du populisme s'inscrit dans la continuité des théories classiques (non seulement de la théorie structurelle-fonctionnaliste mais également de l'école “ développementiste" [Cardoso \& Faletto, 1969]) qui considèrent le populisme des années 30-50 comme un phénomène transitoire, voué à disparaître avec l'avènement des sociétés industrielles (Germani) ou avec le passage de la constitution d'un marché interne au capitalisme dépendant (Cardoso \& Faletto). La réapparition de ce type de mouvement durant les années 80-90 pourrait aussi être interprétée comme un signe d'échec de la modernisation (économique mais aussi politique) des sociétés latino-américaines, dû notamment au système de valeurs ou culture des grandes majorités migrantes favorisant leur résistance à un changement synonyme de métamorphose des croyances et des orientations vitales. Surviendrait aussi l'adhésion à des mouvements susceptibles de raviver en milieu urbain une culture andine d' origine rurale, une thèse que développent les trois ouvrages principaux traitant du vote pour le parti politique bolivien Conciencia de Patria (CONDEPA) dont il sera question dans cet article.

Sans présenter ces thèses dans le détail (2), il convient de souligner qu'elles ont en commun d'opposer fondamentalement les liens communautaires traditionnels " revitalisés " par CONDEPA en milieu urbain à la modernité illustrée par les valeurs compétitives du marché (Saravia \& Sandoval, 1991: 127), une opposition qui, sans résumer la thèse de Saravia \& Sandoval (3), figure pourtant dans le prologue de leur ouvrage où Mansilla fait allusion à " la réapparition de visions simplifiées du monde (1995 : 12-13) " caractéristiques, selon lui, des zones rurales andines. Ce sont ces représentations “ prémodernes (Mansilla, 1995 : 13) ", qu'on dirait ataviques, parmi lesquelles l'auteur du prologue fait ressortir la valeur attribuée au "collectif" $v s$. " l'individuel " qui, instrumentalisées par CONDEPA, contribueraient à expliquer son succès.

En appliquant encore les théories classiques du populisme au cas concret d'un mouvement néo-populiste, on peut émettre l'hypothèse qu'à l'enracinement d'un système de pensée " traditionnel "induisant un rejet du changement devraient logiquement correspondre des pratiques "traditionnelles" d'ordre clientélaire. Cette clientèle populaire passive agirait tel un seul homme (Freud, 1981 : 162), “ manipulée " par des leaders charismatiques (notion centrale dans le travail pionnier de Germani), ce qui suppose, d'après la théorie de la société de masse, une atomisation du corps social et un isolement des individus dans une société devenue vulnérable aux mouvements totalitaires (Arendt, 1958).

(2) Nous renvoyons aux livres suivants pour plus de détails : Archondo, $1991: 148$; San Martin, 1991: 58 et Saravia \& Sandoval, 1991.

(3) Saravia et Sandoval opposent malgré tout "société capitaliste de consommation " et “ conglomérat populaire" (1991: 31). 
L'irrationalité des bases se verrait enfin confirmée par “ leur absence de conscience de classe " sur laquelle concordent les théories antérieurement évoquées ( $C f$. Moscoso Perea, 1990). Contrairement aux sociétés industrielles, en Amérique Latine, la modernisation n'est pas allée de pair avec l'émergence de groupes ayant une conscience de l'intérêt collectif suffisamment développée. Étant donné le contexte de dépendance de ces pays, les classes sociales n'ont pas réussi à devenir les véritables protagonistes de l'histoire (comme le montre la théorie de la dépendance), ce que Ianni reprend à son compte (Ianni, 1975) lorsqu'il écrit que les mouvements de masse (ou populistes) dépolitisent la lutte des classes.

L'objectif de cet article est d'examiner en peu de pages si deux des hypothèses interdépendantes formulées par les théoriciens du populisme sont vérifiées par l'étude d'un cas de néo-populisme. Les bases de Conciencia de Patria sont-elles dépourvues de conscience de classe et de préférence attachées à des valeurs traditionnelles ?, ce qui impliquerait une perception du social en termes de reproduction des inégalités [selon le paradigme de Bourdieu qui limite les probabilités de changement social (4)]. Pour répondre à cette question, nous nous intéresserons au lien qui unit le leader de ce parti, Carlos Palenque Avilés, à son électorat (5) ainsi qu'aux différents mécanismes d'identification impliqués. Dans une première partie, nous verrons comment ces mécanismes donnent lieu à la construction d'un sujet populaire antagonique défini en même temps par opposition et par identification. Dans une seconde partie, nous tâcherons de caractériser le collectif abstrait de peuple en montrant qu'il implique l'émergence de nouvelles identités urbaines ayant pour fondement les droits des individus.

\section{LA CONSTRUCTION D’UN SUJET POPULAIRE ANTAGONIQUE}

La théorie post-marxiste qui a Laclau (1977) comme principal représentant introduit un changement substantiel dans l'étude du populisme en commençant à prendre en compte la subjectivité des acteurs. Elle inscrit, d'une part, le populisme dans la problématique des idéologies qui ont pour fonction de " constituer l'individu comme sujet " sous l'effet d'un discours, d'autre part, dans la problématique de l'identification. Pour le sociologue argentin, ce qui constitue l'identité populaire, est principalement "une relation d'opposition" construite, dans le cas de CONDEPA, à la suite d'un événement majeur : la fermeture (6) par l'État des médias de Carlos Palenque [Radio Télévision Populaire (7)] donnant lieu à la protestation d' environ cent mille personnes

(4) Perspective que propose la sociologue Vega-Centeno dans son étude de l'APRA (1991).

(5) Lien n'ayant pas été suffisamment étudié par les chercheurs qui travaillent sur la notion de charisme, d'après le sociologue Mann (1993).

(6) Cette seconde fermeture de RTP (la plus importante) se produit à la suite de la diffusion d'un programme en direct auquel participe depuis la clandestinité un célèbre narcotrafiquant, Roberto Suárez, qui compromet le gouvernement de Paz Estenssoro dans le trafic de drogue. Palenque est alors accusé de faire l'apologie du délit (Archondo, $1997:$ 3).

(7) Ces médias se composent principalement de radio Métropolitaine (qui sort sur les ondes en 1980) et d'une chaîne de télévision (la quatrième chaîne) que Palenque acquiert en 1985. 
qui se considèrent injustement privées des seuls médias leur offrant un droit à la parole (cette privation est du reste perçue comme un châtiment). L'ampleur des mobilisations s'explique à première vue parce que dès 1980, dans son programme phare "La Tribune Libre du Peuple ", Radio Métropolitaine permettait aux individus d'origine modeste de venir raconter leurs problèmes et inquiétudes quotidiennes, de solliciter une aide ou de dénoncer certaines injustices (8). Palenque les recevait, les écoutait, prenait position, les encourageait et critiquait l'insensibilité du gouvernement.

Au terme d'une première protestation populaire mise à profit par le communicateur social et son entourage, Conciencia de Patria est fondé le 21 septembre 1988. La fermeture de RTP a un double effet : elle favorise l'émergence d'un leader charismatique (9) et, corrélativement, d'un "Nous" collectif (le "peuple") se construisant par identification, c'est-à-dire, tel que nous avons défini ce processus (Alenda, 2001a, à paraître), par " transfert d'une valeur affective sur un objet permettant de donner une certaine cohérence à la réalité perçue par les acteurs ". Le " nous " populaire existe d'abord à travers RTP, puis à travers CONDEPA, et il a comme principe de cohésion Palenque lui-même.

\section{1. Les mécanismes de l'identification : la relation métonymique entre le "peuple" et RTP}

\section{1. 1. La fermeture de RTP et l'émergence d'une "famille populaire"}

L'exaltation émotionnelle que suscite la fermeture des médias renforce l'identification du peuple au leader converti en victime d'un gouvernement antipopulaire. Un nous collectif voit le jour défini par Palenque comme "la famille" populaire :

“Vous avez pleuré et moi aussi j'ai pleuré, j’ai pleuré, j'ai pleuré d'impuissance, vous aussi vous avez pleuré d'impuissance face à l'injustice. Moi aussi, face à l'injustice... Nous avons pleuré ensemble sur la place San Francisco parce qu'il n'y avait plus aucune chance d'obtenir la réouverture du Système RTP, aucune chance, nous avions observé une grève de la faim à laquelle nous avons seulement fait une parenthèse pour nous rendre sur la place San Francisco afin de dire quelques phrases à la famille pour ensuite y retourner et poursuivre notre grève de la faim. " (Palenque, discours du $1^{\text {er }}$ septembre 1988)

Dès lors, Palenque a recours à l'analogie entre sa famille et la famille populaire, ce qui favorise son identification à RTP qui en vient à incarner le peuple. Archondo écrit à ce sujet (1991: 137) :

"La relation métonymique entre le peuple et RTP se produit par la médiation de la famille. Tous les deux forment une communauté dont les membres

(8) Ce que la quatrième chaîne permet aussi lorsque l'émission devient télévisée en 1985.

(9) Notre étude du concept de " charisme " s'inspire des travaux des sociologues Mann et Dobry qui prennent leurs distances par rapport à la "théorie" weberienne de la domination charismatique. $C f$. les articles de Mann (1993), Dobry (1993) ainsi que notre propre thèse pour plus de détails (Alenda, 2001a, à paraître). 
s'aident en permanence. Dans certains cas, RTP assume la paternité du peuple, tandis que dans d'autres, il [le Système] lui doit son existence ".

Dans l'un des discours qui succède à la fermeture des médias, Palenque met en parallèle sa relation conjugale et le lien qui existe entre RTP et le peuple (10). Il se présente aussi métaphoriquement comme " le fils amené à partir [à abandonner RTP] à cause des circonstances, obligé de partir, poussé à se séparer de son peuple, poussé à abandonner le foyer [le " foyer ", la " maison " (11) sont deux métaphores couramment employées pour désigner RTP] dont il s'est toujours senti fier, le foyer du peuple bolivien (12)". Un lien de réciprocité se consolide au sein duquel Palenque occupe accessoirement la place du fils de la communauté dont il est également et principalement le père. Tout comme RTP, il incarne à la fois une chose et son contraire, cette interchangeabilité masquant en fait une relation inégale tout en posant une équivalence entre Palenque, ses médias et le peuple.

Mais l'identification dépend surtout du statut de victime du leader (et par extension de RTP), un statut mis à profit lors de la fermeture des médias puisque sont mis sur un même plan les mesures anti-populaires de relocalisation (13) des mineurs prises par le gouvernement de Victor Paz Estenssoro et le licenciement simultané d'un groupe de travailleurs du Système dû à la fermeture. Ces derniers sont présentés comme des "relocalisés", victimes du même gouvernement insensible :

“... Aujourd'hui, le gouvernement met à la rue 150 travailleurs ainsi qu'un groupe de cadres du Système RTP " (14).

C'est ce même recours à l'analogie qui ponctuera les discours politiques (15) de Palenque qui, au vu des résultats électoraux obtenus par CONDEPA en 1989, se réfère à la " famille agrandie ", mobilisée contre ceux qui avaient voulu " passer sous silence la voix du peuple ". Cette fois, l'analogie s'applique au peuple et à la " nation " ou "patrie".

Les interventions populaires à "La Tribune " faisant suite à la fermeture du Système démontrent l'impact du discours. Un manifestant déclare notamment : “ Nous devons défendre le peuple et nous appartenons précisément à cette famille... " (16). Le

(10) $C f$. pour plus de détails l'ouvrage de Archondo, 1991 : 137-144.

(11) Voir par exemple le discours de C. Palenque prononcéà El Alto le 7 octobre 1994 : “ Merci de ces baisers, de ces accolades, de nous souhaiter la bienvenue, merci, famille condépiste, nous sommes de retour à la maison pour continuer le combat... " [Discurso pronunciado por el Jefe Nal. de CONDEPA, Lic. Carlos Palenque Avilés, en la ciudad de El Alto, a su retorno de Europa, ante unas 3000 personas, el viernes 7 de octubre de 1994 (Palenque, 1995 ; 27)]. Voir aussi " Ce sont comme l'a dit la comadre Mónica nos quartiers, notre ville, où nous sommes nés, la grande maison où vivent nos frères, où vivent nos parents, où vivent nos amis, où vivent des gens comme nous nés sous un même toit " [Discurso pronunciado por el jefe Nal. De CONDEPA, Lic. Carlos Palenque Avilés, en la entrega de la avenida Mariscal Santa Cruz, el sábado 24 de octubre de 1994 (Palenque, 1995 : 31-32)].

(12) Intervention de Carlos Palenque à RTP, août 1988.

(13) Pour plus de détails, voir de Mesa et al. (1997) et Baptista Gumucio (1996).

(14) Intervention de Carlos Palenque à RTP, 7 novembre 1988.

(15) $C f$. les discours du Chef National de CONDEPA du 13 novembre 1994 et du 24 octobre 1994 (Palenque, 1995).

(16) “Tribune Libre du Peuple ”, 7 novembre 1988. 
processus d'identification des masses à RTP va dès lors se construire autour d'une relation symbiotique famille populaire/médias ayant Palenque pour principe cohésif (17). D'après l'ami de jeunesse et proche collaborateur de ce dernier au sein de RTP, Adolfo Paco, les couches populaires voient en lui la personne qui " a lutté pour la justice de ce peuple et qui a toujours tâché d'améliorer le sort des habitants de ce pays ". Selon Paco, la création de CONDEPA résulterait de deux facteurs (18) : la fermeture des médias (identification peuple/médias) et le fait d'éteindre la voix de Palenque (identification plus directement personnalisée à un leader à la fois crédible et charismatique parce qu'il se situe du côté des plus défavorisés). Voici comment un homme qui se présente à " La Tribune " lors de la réouverture d'août 1988 manifeste cette symbiose :

" Cette radio est notre bouche à nous, celle de la population majoritaire, ce n'est pas radio Métropolitaine qui est fermée, ni la quatrième chaîne, mais nous-mêmes. Nous-mêmes, la population en général qui sommes l'État, la Patrie, le drapeau national, nous sommes là [nosotros estantes], nous sommes des habitants. C'est cela que l'on ferme, ce n'est pas vous, Compadre (19). Parce que vous avez fait et faites partie de nous, Dieu en a voulu ainsi (20)".

Les mesures prises contre RTP portent donc atteinte au droit du peuple à l'expression, à son existence même et elles sont mises à profit par le futur leader de CONDEPA qui devient le porte-parole des exclus en leur fournissant " les instruments de la réappropriation de leur propre expérience" (Bourdieu, 1979 : 538) (21). Or il ne suffit pas de permettre aux acteurs d'identifier la source des maux dont ils souffrent pour susciter la protestation. Il faut aussi que Palenque fasse lui-même l'expérience de l'exclusion en occupant la position dominée de victime d'un gouvernement antipopulaire pour que l'ordre social cesse d'apparaitre comme immuable. En effet, la crédibilité du leader comme représentant des intérêts des dominés vs. ceux des dominants se construit par projection, dans le partage d'une expérience commune qui

(17) Dans ce contexte, c'est la relation au Système et à son propriétaire qui l'emporte sur le lien entre les téléspectateurs et le reste de l'équipe de RTP (entre autres, la chola Remedios Loza dont il s'agira plus loin et M. Medina, l'épouse de Palenque qui présentent toutes deux "La Tribune " au côté du leader). Tous ces "symboles" sont dès lors plus ou moins interchangeables — ce que confirmeront les remaniements au sein du parti —, l'important étant que demeure en place le principe cohésif de la " famille" auquel tous doivent leur existence — à commencer par ses proches —, leur ascension sociale... ou leur déchéance. ( $C f$. pour de plus amples détails, Alenda 2001a, à paraitre).

(18) Entretien avec Adolfo Paco, La Paz, octobre 1995.

(19) L'institution du compérage (parenté spirituelle qui dans le monde urbain tend à être instrumentalisée par les leaders politiques pour étendre leurs réseaux sous la forme du patronage politique) permet à Palenque de consolider le lien avec ses partisans d'origine modeste. La dénomination " compadre " s'étendra à tous les condépistes, dirigeants et bases, renforçant la sensation égalitaire d'appartenir à une même " famille ". $C f$. notre thèse de doctorat (Alenda 2001a) pour le détail de l'utilisation du compérage. Sur le patronage politique, voir Lavaud, 1976.

(20) “Tribune Libre du Peuple”, août 1988.

(21) Ceci dit, il serait faux de ne voir en Palenque qu'un "producteur professionnel de discours " dont les dominés seraient "à la merci ", car le leader fait objectivement partie de ceux dont il devient le porte-parole au même titre que chaque militant de CONDEPA investi du rôle de porteparole de la cause populaire. 
renforce l'identification des foules, révélant à elle-même la famille populaire, la " réveillant" [Usted nos ha despertado, Compadre], lui " ouvrant les yeux" [nos ha abierto el ojo] (22).

\section{1. 2. La mise en scène de l'émotion}

Ce qui garantit la permanence de ce lien avec le Compadre et ses médias, est sa reconstruction quotidienne tout au long de la carrière médiatique de Palenque (soit 27 ans). Cette reconstruction s'effectue lors de "La Tribune Libre" par le biais d'une mise en scène de l'émotion suscitée par les enjeux les plus familiers, les plus quotidiens, qui ont justement le pouvoir d'évoquer les sentiments les plus forts, comme le signalaient déjà les chercheurs de l'Université de Michigan :

"we do find that the most familiar issues are also the issues that evoke most strong feeling (Campbell et al., 1960 : 176) ".

Chaque individu se reconnaît dans les problèmes des autres et peut profiter directement des conseils du Compadre. En ce sens, "La ' Tribune Libre du Peuple' en partant de faits locaux, familiaux et individuels extrapole des conclusions d'intérêt général (Archondo, 1991 : 125)" et l'aide apportée par RTP à certains des visiteurs renforce jour après jour le lien communautaire : "La Tribune" est le lieu où le visiteur est à la fois individu et représentant d'une collectivité dont il partage les conditions de vie, une sensation alimentée par l'effet de réalisme de l'image. La déclaration suivante montre comment une auditrice se reconnaît dans les mots d'une femme s'étant présentée à cette émission :

“L'an passé je me suis séparée de mon époux ; j'écoutais la radio et il [Palenque] disait 'comadre' — une autre femme aussi était allée à la radio - ' ' mon époux m'a quittée ', la femme pleurait, et il disait 'comadre, lutte pour tes enfants, élève-les correctement '. C'est pourquoi, lorsque je le [Palenque] vois en personne, je suis émue et je me mets à pleurer (23)".

Enfin, ce qui démontre la force du lien entre le peuple et ses médias est sa survie au décès de Carlos Palenque en mars 1997. En effet, si en 1988, dans un contexte d'intensification des émotions, " faire taire RTP, c'est faire taire le peuple (24) ", le même type d'argument sert à expliquer la fidélité populaire au Système en 1997 (25).

(22) Selon deux militants encore sous l'émotion de la réouverture de RTP, “Tribune Libre du Peuple ", août 1988.

(23) Entretien avec une militante, El Alto, septembre 1995.

(24) Selon les mots d'un commerçant, “ Tribune Libre du Peuple ”, 22 juin 1988.

(25) D'après les résultats d'une enquête par sondage sur laquelle s'appuie en partie notre analyse du vote condépiste. Cette enquête quantitative a été réalisée dans la ville bastion du condépisme : El Alto, dans le cadre de notre terrain de doctorat, avant les dernières élections nationales de juin 1997, auprès d'un échantillon local conséquent de 717 personnes en âge de voter. Les résultats électoraux obtenus par CONDEPA à El Alto sont les suivants : 47\% aux élections générales de 1989, 47,8\% aux élections générales de 1993,55\% aux élections générales de 1997, convertissant cette ville en un laboratoire susceptible d'éclairer les logiques électorales. $C f$. Opiniones y Análisis, Tomo I, 1998 (ces pourcentages ont été calculés sur la base de la totalité des votes valides). 
Les condépistes se perçoivent comme les propriétaires des médias (un électeur déclare : " c'est comme si cette chaîne de télévision m'appartenait "), parce que, selon eux, " ils n'ont pas perdu leur simplicité " et la fidélité à RTP est, dans certaines réponses, synonyme de fidélité au Compadre ("Comment ne verrions-nous pas sa radio et sa chaîne de télévision puisqu'il nous a dit qu'ils étaient à nous, au peuple ? ”). Nos résultats confirment ceux d'une enquête réalisée en 1988 sur la perception de radio Métropolitaine mettant en évidence l'intérêt " néo-communautaire" du public : 32\% des auditeurs y déclarent écouter cette radio parce qu'elle " est populaire et représente le peuple (26) " et seuls $17 \%$ donnent à leur préférence des justifications plus concrètes en affirmant qu'elle " aide les pauvres (27)".

Avant les élections nationales de juin 1997 auxquelles est candidate Remedios Loza (28) à la suite du décès de Carlos Palenque quelques mois auparavant, les Alténiens

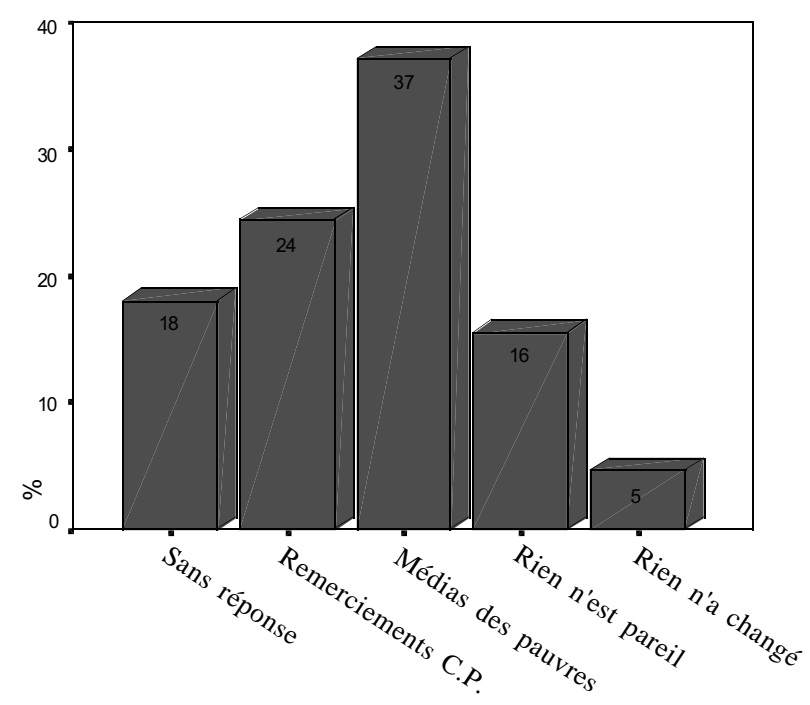

Fig. 1 - Pourquoi (oui ou non) demeurez-vous fidèles à RTP depuis le décès de Carlos Palenque? (question posée aux seuls auditeurs et téléspectateurs de RTP dans la ville de El Alto)

(26) Nous avons additionné les modalités " Elle est populaire " (12\%) et " elle représente le peuple " (20\%) (cf. Saravia \& Sandoval, $1991: 54)$.

(27) Les autres pourcentages sont inférieurs aux deux chiffres cités (excepté la modalité " autres" non spécifiée $=26 \%$ ).

(28) Remedios est une femme de pollera (ou chola) issue du peuple qui participe dès 1969 aux premières émissions radiophoniques de Palenque. Elle devient à l'élection présidentielle de 1989 la première femme députée de sa condition (nous aurons l'occasion d'y revenir). La distinction entre les femmes de pollera et les autres femmes - toutes deux d'origine modeste - repose sur le vêtement en tant que marqueur d'une identité sociale plus ou moins discriminée, les cholas se situant en bas de l'échelle de stratification en cumulant deux " handicaps " : le fait d'être des femmes et d'avoir une origine indienne marquée par leur vêtement. Celui-ci se caractérise par la superposition d'une ou 
qui se déclarent fidèles à RTP (29) se répartissent en deux groupes principaux : ceux qui affirment leur appartenance à une famille populaire (+ : " parce que RTP est le peuple " $=37 \%$ ) et ceux qui entendent témoigner leur reconnaissance au chef défunt de la communauté (+: “en remerciement à Carlos Palenque " $=24 \%$ ). En 1997, l'identification à Radio Télévision Populaire semble donc bien survivre à la disparition du Compadre.

\section{2. Les mécanismes de l'identification : la relation métonymique entre le peuple et CONDEPA}

En même temps que la fermeture de RTP naît le mythe d'un parti et d'un candidat à la présidence issus de la volonté du peuple, un mythe entretenu par Carlos Palenque s'auto-identifiant au peuple [" le peuple dont nous faisons partie "(30)]. Dès sa création, CONDEPA se définit comme un mouvement caudilliste construit sur le rapport d'équivalence entre le peuple et Palenque converti en leader éponyme du mouvement. À l'occasion de la première fermeture de RTP, l'un des employés des médias enjoint le Compadre à être candidat à la présidence de la République :

“Maintenant tu dois être le candidat à la présidence et cette nuit, nous entamons une grève de la faim et naît le palenquisme en Bolivie (31)".

Or ce n'est que lors de la seconde réouverture de RTP que Palenque déclare en démontrant ses talents rhétoriques " accepter la volonté de son peuple et se proclamer candidat (32) " dans un discours égalitaire (33) valorisant les plus démunis (notamment à travers l'idéalisation de la " sagesse populaire ") :

“La souveraineté réside dans le peuple et le peuple a choisi son candidat ce jour-là. Et c'est sur la place San Francisco même que vous et moi avons pleuré, nous avons fait la fête parce que, d'une part, nous avions un candidat qui devait accomplir une mission, d'autre part, le peuple avait récupéré ses médias et il fallait danser, il fallait chanter, il fallait faire la fête. Et il en fut ainsi. Nous avons fait la fête ensemble au cours d'un rassemblement historique incroyable et à l'heure où je vous parle, la tâche de mobilisation du peuple continue bien que ce parti politique ou mouvement politique, comme il convient davantage de l'appeler, n'ait

plusieurs jupes et par le port du chapeau melon et du châle à la différence des femmes de vestido vêtues à la mode occidentale, voire d'un pantalon.

(29) Le détail des résultats est le suivant : $37,8 \%$ de réponses positives contre $8,1 \%$ de réponses négatives et $7,7 \%$ de sans-réponse (pour cette question, seuls 384 individus ont été retenus sur une base totale de 717$)$. $C f$. notre enquête par sondage.

(30) Carlos Palenque, discours du $1^{\text {er }}$ septembre 1988.

(31) Employé de RTP, La Paz, 5 juin 1988.

(32) “ J'accepte la volonté de mon peuple et me proclame candidat... mes chers compadres, moi au moins, j'ai toujours écouté votre volonté. Je n'ai jamais agi seul dans ma vie, je vous ai toujours demandé conseil par téléphone parce que le peuple est sage. Cela fait vingt ans que j'apprends [aprendido] la sagesse populaire, que j'ai voulu servir mon peuple depuis radio Métropolitaine et depuis cette chaîne de télévision ", Carlos Palenque, discours du 13 août 1988.

(33) Il déclare à ce propos le 22 juin 1988 : "Nous sommes tous égaux, nous travaillerons d'égal à égal, nous émergerons tous, nous nous projetterons tous dans l'avenir, tous nous vaincrons !". 
pas de nom mais il a un capital merveilleux qui est son peuple." (Palenque, discours du 1er septembre 1988).

Il s'agit bien ici du mythe fondateur d' un mouvement que le Compadre n'accepte de diriger qu'en tant qu'instrument au service de la communauté. Le peuple en est la définition même, le principe légitimant l'action. On peut dire que le mythe remplit ici son rôle de "véhicule d'expression des émotions (Álvarez Junco, 1994 : 222) " et contribue à donner la sensation de démocratie participative en rendant crédible la démocratie directe comme l' atteste l' euphorie des manifestations populaires de soutien à Palenque. En effet, les premiers militants se souviennent d'avoir eu la sensation d'être les protagonistes d'un événement historique perçu comme l'amorce du retournement de l'ordre social au bénéfice de la "majorité" protestataire, fondant ainsi leur reconnaissance sur le nombre. Ils ne font, en ce sens, que reprendre les propos du Compadre qui justifie sa lutte par un argument de sens commun au fondement de l'idée de démocratie directe : "la majorité doit l'emporter sur la minorité ". Voyons comment ces militants se font l'écho des propos du leader en transformant la mobilisation pour la réouverture de RTP en une lutte pour la " démocratie " entendue comme le droit d'expression des couches populaires :

“ Compadre, je veux insister Compadre, vous l'avez dit vous-même, c'est la majorité [las mayorías] qui commande, nous avons tellement lutté pour la démocratie, Compadre, et elle nous a coûté la démocratie, nous avons lutté pour l'obtenir dans les médias. Maintenant, nous avons la démocratie, malheureusement ils sont en train de la détourner mais c'est avec la démocratie que nous allons atteindre nos objectifs, Compadre" (34).

La parole apparaît comme l'élément fondateur de cette “ idéologie de la démocratie pure, du peuple devenu pouvoir ou du pouvoir devenu peuple (Furet, 1978 : 92) ". Dès la fermeture de RTP, les couches populaires s'approprient les mots du Compadre qui forgent un sujet politique en l'élevant contre une vague antithèse (" ils ") à laquelle continuent de faire allusion certains électeurs interrogés en 1997 sur l'éventuelle disparition de CONDEPA. Un condépiste répond notamment: "Nous continuerons contre qui que ce soit" [Seguiremos adelante contra quien sea]. À l'évidence, le discours de Palenque influe sur la construction de ce sujet populaire pour une raison pouvant être liée au fait que ce système de représentations, s'il dit bien que le pouvoir peut être pris, dit également qu'il ne saurait l'être que par un représentant du peuple suffisamment crédible et de ce fait légitime pour permettre la victoire (étant donné que le pouvoir n'est pas vacant). On comprend par conséquent que la démocratie ne puisse être conçue que sous une forme directe, “ le peuple ne pouvant par définition aliéner ses droits à des intérêts particuliers (Furet, 1978 : 222)".

(34) “Et nous ne permettrons pas qu'on nous gouverne, nous gouvernerons, nous ne permettrons plus qu'ils nous manœuvrent, nous les manœuvrerons, la majorité doit l'emporter sur la minorité, compadres, et non la minorité sur la majorité, compadres. C'est ce que nous allons obtenir ensemble... vous et nous !", Discours de Carlos Palenque (fermeture de RTP), La Paz, 7 novembre 1988. Nos remerciements à Rafael Archondo pour nous avoir permis l'accès à ces discours. 
Le glissement de RTP à CONDEPA s'effectue dès la seconde réouverture des médias où les foules ne scandent plus " Vive RTP " mais " Palenque, futur Président ", "Compadre Palenque, le peuple est avec toi " ou encore " Palenque à la présidence ", des slogans forgés et transmis à leurs bases par les dirigeants expérimentés de la Fédération des Organisations de quartiers (FEJUVE) qui intègrent très tôt le mouvement (35). Suivant le même processus observé pour RTP, une relation symbiotique se construit cette fois entre le peuple et son instrument politique : CONDEPA, dans laquelle Palenque occupe toujours la place centrale. C'est aussi par l'argument d' un vote conçu comme l'expression d'une néo-communauté populaire (OUI : " parce que CONDEPA est le peuple" = 39\%) que les individus sondés avant les élections nationales de 1997 expliquent majoritairement (et toutes opinions politiques confondues) (36) les raisons pour lesquelles Remedios Loza recevra le soutien de l'électorat (37):

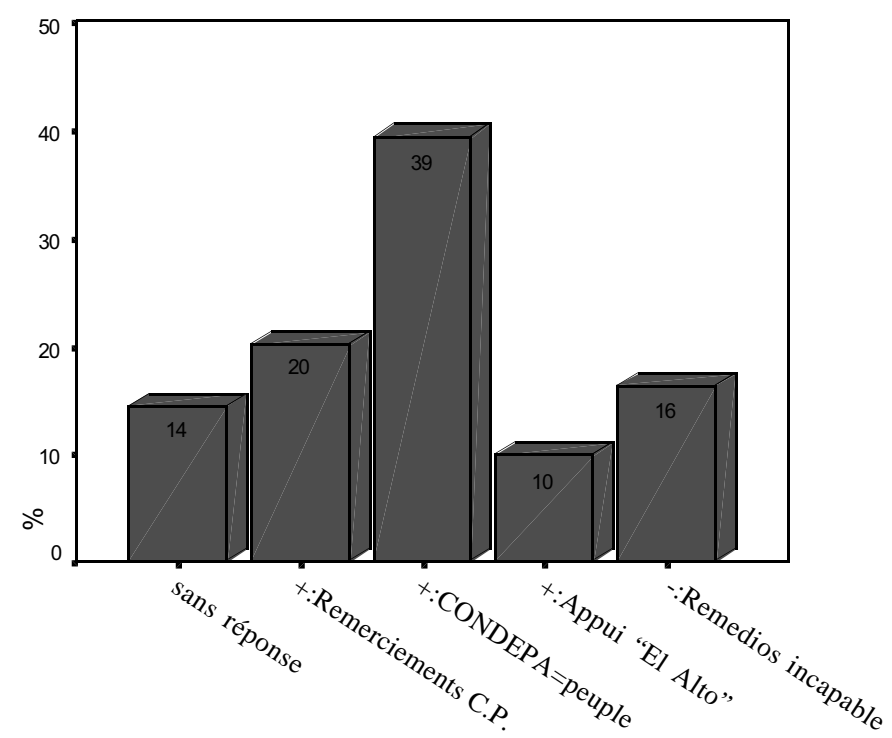

Fig. 2 - Pourquoi (oui ou non) pensez-vous que Remedios Loza recevra le soutien de l'électorat dans la ville de El Alto?

(35) D'après le témoignage de Genaro Torres recueilli par Archondo : “ Le slogan n'était plus ' vive RTP' mais ' Compadre Carlos, futur Président '. C'est ce que j'enseignai, je donnai des instructions à tous les gens qui me suivaient [mi gente], pour qu' ils fassent circuler l'information auprès de chaque organisation, chacune faisant la queue pour commencer la manifestation ". G. Torres signale aussi le slogan : "Compadre Palenque, le peuple est avec toi ". Le témoignage de Elsa Alcazar confirme celui de Torres : "On nous disait de dire : Palenque à la présidence" (propos recueillis par Rafael Archondo).

(36) Notons cependant que la tendance à voter pour CONDEPA est la plus forte pour la modalité "CONDEPA=peuple " ( $c f$. pour plus de détails , Alenda, 2001a, à paraître).

(37) À la question, "Pensez-vous que Remedios Loza recevra le soutien de l'électorat ?", $73,2 \%$ ont donné des réponses positives contre $18,8 \%, c f$. notre propre enquête par sondage. 
Nous avons vu quels étaient les mécanismes de l'identification populaire se résolvant dans la chaîne d'équivalences suivante $:$ peuple $=\mathrm{RTP}=\mathrm{CONDEPA}=$ Palenque. Tâchons maintenant d'étudier la fonction de ce processus d'identification.

\section{3. Identification et émergence d'une identité collective positive}

La valorisation du peuple contribue à expliquer l'identification totale. En effet, RTP ne peut pas être considéré comme un facteur de simple " conservation " (MacLuhan, 1968) ou de " renforcement" (Klapper, 1960) des conditions sociales existantes. Le message est avant tout crédible parce que cette réalité médiatisée répond justement à une quête de sens : le besoin d'être reconnu, de se sentir intégré, un sens transmis par un discours sur les pauvres, pour les pauvres, ayant des effets structurants. Avant de valoir pour CONDEPA, la formule suivante des chercheurs de l'Université du Michigan s'applique bien à RTP :

“ The political parties provide one organizing dimension that seems accessible to persons of very impoverished political understanding " (Campbell et al., 1960 : 253).

C'est pourquoi on n'observe aucun phénomène de sélectivité portant sur le choix des messages, lesquels ne sauraient être en désaccord manifeste avec les valeurs et les normes des plus défavorisés quelles qu'elles soient puisque les médias s'adressent à des secteurs sociaux aspirant à être intégrés, dont ils font, de surcroît, les véritables protagonistes du changement (en développant l'estime de soi des couches populaires). Les valeurs diffusées (la solidarité, la réciprocité ou encore la persévérance dans l'effort), ainsi que les croyances (principalement celle d'un retournement de l'ordre social au bénéfice des pauvres), parce qu'elles émanent de représentants crédibles pour le peuple pour les raisons antérieurement évoquées, sont susceptibles d'influencer les comportements et de modeler les identités (38), notamment par le biais d'un discours moralisateur, une "éducation morale", notion que Durkheim présente comme la contrainte la plus subtile et efficace dont dispose la société pour ses membres (Boudon $\&$ Bourricaud, 1982 : 121). Cette éducation vise principalement à la préservation de l'harmonie familiale suivant un certain nombre de règles prescrites (condamnation de toute forme de violence conjugale et familiale), dont il est difficile de mesurer exactement l'impact, mais qui semblent plutôt bien accueillies par les foules (i.e. les personnes venant témoigner à "La Tribune " prennent acte des réprimandes que leur adresse le Compadre (39) sans se sentir agressées ; la relation n'est donc pas désavantageuse pour l'une des parties).

(38) Au sujet de l'influence du parti sur les comportements, voir notre thèse de doctorat où nous expliquons la plus grande tolérance des hommes vis-à-vis de l'émancipation de leur épouse, une tolérance influencée par l'attitude du Compadre envers sa propre épouse. Au sujet de l'influence sur les constructions identitaires collectives en milieu urbain, voir aussi Alenda (2001b ).

(39) Citons par exemple le cas d'un homme auquel la famille était venue reprocher à " La Tribune ", entre autres, le fait qu'il ne veuille pas prendre en charge sa mère âgée. Quelques jours plus tard, l'individu rappelé à l'ordre par le Compadre se présentait à "La Tribune " afin de démentir [vengo a desmentir] d'autres accusations dont il avait été l'objet de la part de sa famille. Le Compadre l'écouta puis lui fit la morale avec un discours tombant sous le sens : votre mère vous a protégé/ vous vous devez 
En établissant un lien social (en permettant une communication imaginaire entre ceux d'en bas et ceux d'en haut), la valorisation joue un rôle intégrateur, ce qui transparaît à travers les propos d'un condépiste d'origine rurale :

"Parce que vraiment, avant, aucune sorte de travailleur, ni même le plus humble, le paysan, ne pouvions avoir ce privilège de parler dans ce micro, mais aujourd'hui grâce à vous Compadre, et c'est ce que je veux que sache toute la population bolivienne en général, nationale, grâce à votre initiative, tous nous pouvons utiliser ce micro, peut-être que notre voix peut se faire entendre, que cela soit bien ou mal interprété (40)".

En synthèse, la fermeture de RTP donne lieu à l'émergence d'une communauté imaginaire construite sur un principe d'exclusion qui permet au " peuple " d'émerger contre l'ordre établi. L'antagonisme de classe mis en évidence par Laclau s' inscrit aussi dans cette logique d'identification où l'identité populaire " nous/famille populaire " se trouve renforcée par l'opposition aux “ autres " (c'est-à-dire tous les gouvernements anti-populaires), le peuple ne devenant un sujet agissant autonome que constitué en contre-pouvoir. Cependant, l'idéologie de CONDEPA est aussi fortement intégratrice puisque cette néo-communauté est paradoxalement fondée sur un principe d'égalité, sur des droits inscrits dans une idéologie de la démocratie pure faite d'une série d'équations imaginaires : peuple $=\mathrm{RTP}=\mathrm{CONDEPA}=$ Palenque. Enfin, le sentiment $\mathrm{d}$ ' appartenance à une identité générique est suscité par un personnage d'autant plus crédible et charismatique (41) qu'il est à son tour victime de mesures injustes en n'ayant guère de concurrents sur le terrain de l'aide sociale... Pour l'un des organisateurs des mobilisations de 1988, le pouvoir qu' a Palenque de susciter au moins en partie la protestation tient dans le fait qu'“ il était connu, [qu']il avait aidé les dépossédés et [qu']il avait fait un travail humanitaire et social (42)".

Posons d'une part que l'influence du discours de revalorisation est proportionnelle à la discrimination (ce que nous tâcherons de montrer dans la seconde partie de cet article à travers l'étude du comportement électoral du sous-groupe des cholas); d'autre part, que certaines structures sociales sont susceptibles d' accroître cette influence (notamment dans le cadre de rapports concurrentiels en milieu urbain). Dans les pages suivantes, il s'agira de caractériser le concept vague de peuple (en rompant avec sa représentation en termes de totalité homogène) et de montrer qu'il existe plus d'une logique du vote.

réciproquement de lui apporter aide et soutien et de ne pas la laisser à la rue. L'homme revint à son démenti tout en reconnaissant ses torts : " Très bien, Compadre, je reçois toutes les recommandations que vous me faites, Compadre, merci ". Cas retranscrit par Archondo, 1988.

(40) Déclaration d'un manifestant à "La Tribune Libre du Peuple ”, 16 août 1988.

(41) Nous entendons par-là en peu de mots que le caudillo a le pouvoir de délimiter l'univers des possibles et de remettre en cause la légitimité de l'ordre dominant.

(42) Témoignage de Genaro Torres recueilli par Archondo. Deux ans avant l'achat par le Compadre des droits de fréquence de radio Métropolitaine, une enquête montre qu'il est devenu "le personnage radiophonique le plus connu des migrants dont 90\% déclarent l'avoir écouté " (Archondo, $1991: 87)$. 


\section{LE CONCEPT DE PEUPLE OU L'ÉMERGENCE DE NOUVELLES IDENTITÉS URBAINES}

\section{1. RTP ou la communauté réinventée}

Dans son ouvrage sur CONDEPA au sous-titre évocateur de "La résurrection métropolitaine de l' ayllu ", Archondo entend montrer que Palenque réussit à " soulever les démons intérieurs des aymaras " en revitalisant la communauté andine en milieu urbain (43), une thèse qui va dans le sens des théories classiques du populisme.

Pour Archondo, le public deRTP — et parextension les partisans deCONDEPA — serait constitué de déracinés d'originerurale, nostalgiques d'anciennes formes d'organisation indienne. Ceci expliquerait le lien inconditionnel au leader — garant de la pérennité d'une culture — et à sesmédias, que les migrants sont aussi “ les premiersàchoisir(Archondo, 1991 :89)". Archondo nuance toutefois certaines de ses conclusions en citant les résultats d'une enquête qui montre que " la préférence urbaine se rapproche des programmes bilingues en espagnol et en aymara et s'éloigne de ceux qui n'utilisent que l'une des langues mentionnées (...) tandis que 72\% des paysans écoutent des radios qui n' utilisent que l'aymara, $67 \%$ des migrants urbains sondés optent pour un contenu bilingue (...)" (44).

À l'inverse de la thèse de Archondo, d'autres travaux font apparaître les massmédias comme un ferment d'acculturation très actif parce que les messages qu'ils véhiculent, auxquels les formes culturelles traditionnelles résistent d'ailleurs mal, sont en grande partie élaborés dans les sociétés industrielles avancées (Schramm, 1964). Ces études insistent sur le fait que les médias provoquent un intérêt pour des aspects de la vie sociale jusqu'alors totalement ignorés, favorisant l'intégration, et par conséquent l'atténuation des spécificités culturelles, en un mot l'uniformisation.

Dans notre enquête, même si l'écart entre les pourcentages demeure modeste, il apparaît que "Métropolitaine " et la quatrième chaîne tendent à être plus appréciées des migrants nés dans les zones rurales du département de La Paz, en général aymaras, que des individus originaires de El Alto (45). Ces chiffres sembleraient donc, à première vue, valider la thèse de Archondo, d'autant plus fortement que les intentions de vote pour CONDEPA confirment cette tendance (Tab. 1). Pourtant, l'explication du vote pour CONDEPA par l'origine rurale du public de RTP est loin d'être évidente : notre enquête montre que les effets du lieu de naissance sont soit complètement annulés par le fait de préférer la quatrième chaîne (Tab. 2), soit atténués par le fait d'écouter de préférence radio Métropolitaine (46).

(43) L'auteur justifie sa thèse en se référant à certaines déclarations du leader de CONDEPA suggérant l'idée d'une résurrection métropolitaine de l'ayllu, déclarations que, selon nous, Archondo prendre trop au pied de la lettre (Archondo, $1991: 72-73$ ).

(44) CIPCA (1976-1977). Cf. Archondo, 1991 : 79. Rappelons que les émissions de RTP les plus suivies ne sont justement pas celles qui font un usage exclusif de l'aymara.

(45) Nous obtenons à la question portant sur les préférences radiophoniques 32,4\% d'auditeurs d'origine rurale contre $20,4 \%$ d'origine urbaine. L'écart se resserre dès lors qu'il s'agit des goûts télévisuels (29\% contre $22,9 \%$ ), ce qui pourrait refléter la moindre importance des programmes en langue aymara sur les écrans (pour le détail de ces résultats, $c f$. Alenda, 2001a, à paraître).

(46) La tendance observée est en effet la même pour la variable : "Quelle est la radio que vous préférez écouter?" ( $c f$. Alenda, 2001a, à paraître). 
Tab. 1 - Intentions de vote de la population de El Alto à l'élection présidentielle de 1997 (\%) en fonction du lieu de naissance.

\begin{tabular}{|l|ccc|}
\hline & CONDEPA & Autres Partis & Total (Effectifs) \\
\hline El Alto & 43,3 & 56,7 & $100(402)$ \\
Dépt. de La Paz (zone rurale) & $\mathbf{6 6 , 7}$ & 33,3 & $100(210)$ \\
Hors du dépt. de La Paz & 37,9 & 62,1 & $100(103)$ \\
Sans-réponse & 50 & 50 & $100(2)$ \\
Total (Effectifs) & $49,4(354)$ & $50,6(363)$ & $100(717)$ \\
& & & \\
\hline
\end{tabular}

Tab. 2 - Intentions de vote de la population de El Alto à l'élection présidentielle de 1997 (\%) en fonction du lieu de naissance et de la chaîne de télévision préférée.

Population 1 : La chaîne de télévision préférée est la quatrième chaîne (Système RTP)

\begin{tabular}{|l|ccc|}
\hline & CONDEPA & Autres partis & Total (Effectifs) \\
\hline El Alto & $\mathbf{8 3 , 7}$ & 16,3 & $100(92)$ \\
Dép. de La Paz (zone rurale) & $\mathbf{8 2}$ & 18 & $100(61)$ \\
Hors du dépt. de La Paz & $\mathbf{8 5}$ & 15 & $100(20)$ \\
Total (Effectifs) & $83,2(144)$ & $16,8(29)$ & $100(173)$ \\
\hline
\end{tabular}

Population 2 : La chaîne de télévision préférée est n'importe quelle autre chaîne

\begin{tabular}{|l|ccc|}
\hline & CONDEPA & Autres partis & Total (Effectifs) \\
\hline El Alto & 30,7 & 69,3 & $100(277)$ \\
Dépt. de La Paz (zone rurale) & $\mathbf{5 9 , 4}$ & 40,6 & $100(128)$ \\
Hors du dépt. de La Paz & 24,3 & 75,7 & $100(74)$ \\
Sans-réponse & 100 & - & $100(1)$ \\
Total (Effectifs) & $37,5(180)$ & $62,5(300)$ & $100(480)$ \\
\hline
\end{tabular}

Ces résultats font apparaître l'influence socialisatrice des médias auxquels l'électorat de CONDEPA s'identifie quel que soit son lieu de naissance sans que cette socialisation politique puisse être tenue pour un simple ferment d'acculturation à moins de considérer cette dernière comme un effet inhérent aux règles commerciales de l'activité médiatique. Même si l'on peut supposer que les téléspectateurs de RTP sont " acculturés " parce qu'ils suivent des émissions qui prétendent à l'évidence être vues et écoutées par un large public dont les spécificités culturelles importent peu, comment expliquer qu'ils préfèrent les programmes de la quatrième chaîne (notamment les 
feuilletons télévisés ou telenovelas) à produits au moins équivalents sur d'autres chaînes ? L'argument d'une "étiquette" (47) attachée à ces programmes [celle de médias qui " appartiennent au peuple, aux pauvres (48) "] nous semble éclairer ici de manière convaincante l'identification à une néo-communauté qui réussit à fidéliser le public, de même que l'identification à une famille politique contribue à motiver le vote pour CONDEPA.

Les condépistes interrogés définissent cette néo-communauté comme un espace local, urbain plutôt que rural, dans lequel se trouvent concentrés les votants. Comme le déclare une électrice en 1995 : “ la majorité des gens de la ' hoyada' [La Paz], d'ici, de la ville de La Paz est avec CONDEPA ”. Il serait donc tout aussi inexact de dire que RTP atténue les spécificités culturelles que de défendre l'idée d'une revitalisation de cellesci. Disons plutôt que les médias recréent un sentiment d'appartenance à un monde ayant cessé d'être rural en renforçant les liens de ce qui se présente, à travers les propos d'une militante alténienne, comme une communauté urbaine :

“J'écoute radio policial (49) parce que je vois directement les images. Parfois, nous ne pouvons pas nous rendre à la ville pour des raisons économiques, la télévision et la radio nous informent dans le détail, sans mentir, parce que les autres radios mentent, elles n'informent pas bien, mais avec radio Métropolitaine et Telepolicial que nous regardons le soir, nous pouvons voir qui est mort, de quelle manière, parfois nous retrouvons nos proches en regardant la télévision (50)".

Si ce type d'identification à une communauté territoriale est susceptible de refléter un sentiment d'appartenance caractéristique d'une culture paysanne (Demélas, 1992 : 249) (51), il est avant tout reconstruit dans un contexte particulièrement précaire, au sein duquel la compétition est forte : la ville de El Alto (52). Voyons comment la perception de l'inégalité des chances d'ascension sociale s'y trouve amplifiée, tout particulièrement pour les électrices de pollera.

(47) Le mot est de Adolfo Paco (entretien avec A. Paco, 1995).

(48)“ Nous demandons qu' on nous rende la radio parce que la radio est au peuple, elle est aux pauvres", réponse d'un manifestant interrogé par Saravia le 22 juin 1988 sur l'enjeu des mobilisations. Nous remercions Archondo d'avoir mis à notre disposition cette enquête.

(49) Chronique de faits divers particulièrement dramatiques. Le " Metropolicial " qui fait selon Archondo son apparition en même temps que radio Métropolitaine est l'un des programmes qui contribue à étendre la renommée de la radio, puis de la quatrième chaîne en devenant le "Telepolicial ".

(50) Entretien avec une militante, El Alto, 1995.

(51) Pour Favre, " le sentiment d'appartenance des Indiens se réduit traditionnellement à la communauté locale " (1976: 73-86).

(52) El Alto apparaît en 1999 comme la grande agglomération métisse (59\% de la population de cette ville y est née d'après le dernier recensement) la plus pauvre de Bolivie (soient $73 \%$ des foyers, un indice qui demeure constant depuis le dernier recensement de 1992). (cf. Ministerio de desarrollo humano, 1993). Une comparaison de cet indice avec son équivalent pacénien donne une idée de son ampleur : en 1992, La Paz ne comptait que 44\% de foyers pauvres. 


\section{2. Identification partisane et individualisme des cholas urbaines}

L'étude du comportement électoral des femmes de pollera nous permet d'appréhender une identité ne possédant de marqueurs distinctifs que pour la population féminine. Elle peut, en outre, nous aider à préciser ce que recouvre la vague notion de " peuple" puisque les cholas semblent incarner pour les couches populaires l'une de ses expressions la plus pure comme le démontrent les propos d'un manifestant contre la fermeture de RTP, venu saluer l'investiture de Remedios Loza lors de l'élection présidentielle de 1989 : " nous sommes issus de la pollera".

En 1989, pour la première fois dans l'histoire de la Bolivie, une chola est élue au poste de députée. Cette élection est emblématique du fait de la discrimination dont souffrent les femmes, d'autant plus forte qu'elles sont de pollera. Au cours de la campagne présidentielle s'opère aussi un processus de revalorisation culturelle (53) qui ne peut que favoriser l'identification des cholas à CONDEPA.

L'ascension de Remedios, femme artisan d'origine modeste fait rêver les militantes et les encourage car :

“ Remedios, pour ce qui est des femmes du peuple, a dépassé [ha superado] [sa condition]. Grâce à elle, nous aussi, aujourd'hui, les femmes, certaines femmes, pas dans leur totalité, nous travaillons dans les institutions, comment dire, à la mairie, avant cela n'arrivait pas. C'est grâce à elle. Et toutes les femmes, nous devons nous préparer de la sorte, parce que nous ne pouvons pas rester en arrière, parce qu'il devrait y avoir en ce moment de nombreuses Remedios qui se préparent de la sorte (54)".

Pour les militantes, apprendre à faire de la politique revient à imiter la Comadre, à vivre son “élévation" alors même qu'elles occupent les niveaux les plus bas dans l'échelle des emplois municipaux. Le modèle de réussite-Remedios balise en quelque sorte l'ascension sociale de ces femmes en leur fournissant un code de comportement. La chola incarne la supérieure hiérarchique de ses "filles " qui agissent à la mairie à l'image de leur "mère", reproduisant elle-même au Parlement le travail social de Palenque (55) :

“ À la mairie, je défends la femme, je défends les enfants, je défends les gens qui sont battus, et les gens viennent me chercher, et vous savez comment les gens l'interprètent de ma part ?... comme si j'étais une de plus comme la comadre Remedios Loza, c'est ainsi qu'ils me considèrent, ils viennent me chercher quel que soit le problème. Feli [collègue de

(53) D'après un des ouvrages sur CONDEPA : “Avec sa postulation, les condépistes prétendent montrer l'image d'une double revendication pour le genre féminin, d'une part en finir avec la discrimination contre la femme et d' autre part mettre un terme à la discrimination ethnico-culturelle de la femme. Lors de la campagne électorale, ce dernier facteur contribue à la tâche de Revalorisation Culturelle et à accroître la fierté des femmes identifiées " (Saravia \& Sandoval, 1991 : 173).

(54) Entretien avec la comadre Trinidad, employée municipale à El Alto, 7/12/1996.

(55) Remedios présidait au parlement la "Commission de la femme " où elle recevait les plaintes quotidiennes des femmes de sa condition, entre autres, maltraitées par leur époux. Comme à RTP, la députée orientait, apportait un soutien moral ainsi qu'une aide juridique. 
l'interviewée] peut en témoigner, ils viennent me chercher. J'attire des gens, ils viennent, bien qu'ils aient divers problèmes, tu sais que lorsque quelqu'un a des problèmes, le seul fait d'en parler, et c'est réglé, alors je leur permets de me raconter, je leur dis : 'tu dois faire ça, comme ça et cette autre chose pas comme ça', le fait d'orienter les gens suffit, ils viennent m'appeler comme si j'étais la mère (56)".

Tout en orientant l'action des employées municipales, Remedios prend plus largement part (en touchant de ce fait l'ensemble de l'électorat) à la redéfinition du rôle de la femme de pollera urbaine à quelques mois de la fondation de CONDEPA. Le message féministe de la chola exerce un impact immédiat sur les femmes de sa condition comme en témoignent certains commentaires recueillis en 1997 tels que : “ s'il n'y avait pas eu ce parti, nous serions restées en arrière, dans nos maisons (57) ", se faisant l'écho de ce que Remedios avait déclaré en 1989 :

"Traditionnellement, on a seulement valorisé la femme dans son rôle domestique : en tant que mère et femme d'intérieur. Aujourd'hui, elle est en train de s'intégrer à l'activité productive et professionnelle dans des conditions désavantageuses : elle occupe des postes de moindre niveau de salaire (58) et de responsabilité (59)".

Même si Remedios démontre aux femmes de pollera que leur ascension sociale est possible en leur en indiquant la voie, ceci n'implique pas la fin des inégalités. En d'autres termes, les barrières sociales sont devenues invisibles mais elles n'ont pas pour autant cessé d'exister, ce qu'exprime la députée lorsqu'elle invite les cholas à " se libérer" de leur rôle domestique, sans omettre de préciser que leur intégration socioprofessionnelle se réalisera dans un contexte urbain difficile...

Dans leur ouvrage surCONDEPA, Saravia \& Sandoval présentent l'augmentation des populations pacéniennes et alténiennes comme le résultat des différents flux migratoires responsables de la croissance incohérente et vertigineuse de la ville de El Alto (60), ayant donné lieu à “ l'émergence de nombreux besoins insatisfaits provenant des domaines économique, social et culturel " (Saravia \& Sandoval, 1991 : 19). Dans ce contexte, les auteurs mettent en évidence le nouveau rôle des femmes qu'ils considèrent en partie explicatif du condépisme (Saravia \& Sandoval, 1991 : 30). À El Alto, un nombre important de cholas a été amené à cumuler les emplois précaires afin de pallier le faible revenu d'un époux souvent démissionnaire. Elles se sont trouvées insérées, malgré elles, dans un système à la compétitivité accrue par l'affluence d'une main-d'œuvre non qualifiée en quête d'intégration, El Alto étant située au carrefour du

(56) Entretien avec la comadre Isabel, employée municipale à El Alto, 12/10/1995.

(57) Entretien informel avec une électrice, El Alto, novembre 1996.

(58) Ce qui correspond pour la Bolivie, dans les termes de l'INE, à une “ sous-valorisation du travail féminin, ce qui pourrait être un indicateur de sous-emploi " ( $c f$. Ministerio de planeamiento y coordinación, 1992: 71).

(59) Déclaration de Remedios Loza extraite de l'hebdomadaire El Patriota, semana del viernes 17 al jueves $231989, \mathrm{n}^{\circ} 17$.

(60) Pour plus de détails, nous renvoyons à l'ouvrage de Antezana Villegas (1993 : 321). 
monde rural et urbain et alimentant de ce fait tous les espoirs d'ascension sociale (61) en étant perçue comme une " porte d'entrée sur le marché du travail (Ministerio de planeamiento y coordinación, 1992: 46) ". Or cette “ intégration " n'a pu contribuer qu' au développement incontrôlé de l'économie informelle (62) (artisanat domestique, vente ambulante et autres services en milieu urbain et rural) touchant indistinctement les femmes nées à El Alto et en zone rurale pacénienne.

Remedios Loza vante donc les capacités des cholas en leur attribuant les mêmes chances qu' aux “ autres ". Cependant, elle réaffirme parallèlement l'inégalité face à la réussite sociale, dont la perception ne peut qu'être amplifiée pour trois raisons :

1. Elle est influencée par Remedios, donnée primordiale qui explique l'impact d'un discours ambivalent ne pouvant que stimuler la combativité des femmes de pollera, consolider l'identification à la députée et renforcer corrélativement le lien à Palenque, protecteur indispensable dans un système dont on sait par la députée qu'il tend à exclure les femmes de pollera, ce que la concurrence professionnelle à El Alto se charge de démontrer au quotidien.

2. Elle s'inscrit dans un contexte de grande précarité conduisant une maind'œuvre non qualifiée (essentiellement féminine), en quête d'intégration, à grossir le secteur informel de l'économie. Cette intégration professionnelle affecte, en outre, le comportement des individus dans leur vie conjugale et familiale. Il apparaît en effet que la femme condépiste ne veut plus être assujettie à l'homme (réduite au strict rôle de “ femme au foyer") quel qu'en soit le coût. Elle tient au contraire, pour parler comme Remedios, à “ s'intégrer à l'activité productive et professionnelle ", situation pouvant conduire à une inversion des rôles (les enfants restent à la maison avec leur père tandis que leur mère travaille). Cependant, les maris ne voient en général pas d'un bon œil l'individualisme (63) de leur femme, c'est-à-dire non seulement son autonomisation (puisque celle-ci n' hésite pas à privilégier sa carrière politique), mais également le fait qu'elle assume " "en toute liberté ' ses croyances, ses opinions" (Boudon \& Bourricaud, 1982 : 301), notamment politiques. Suivant cette même logique, de nombreuses électrices de base prendront l'initiative d'aller dénoncer au Compadre ou à Remedios Loza la violence de leur époux, adoptant à l'égard de celui-ci une attitude rebelle, reflet de leur propre autonomisation. C'est ainsi qu' "une idéologie 'individualiste ' peut se développer dans un contexte politique où une classe a l'impression d'être injustement et illégitimement bloquée par le système politique, même dans le cas d'une société 'traditionnelle '" (Boudon \& Bourricaud, 1982 : 305). Il convient de signaler que cette idéologie diffusée par le parti a des effets pervers. Car ce discours centré sur la femme mais aussi sur le couple (Carlos Palenque et son épouse Monica Medina) et la famille tend à encourager la libération féminine en mettant du même coup en péril la cellule

(61) Parce que " tout paysan a plus ou moins intériorisé l'idéologie dominante selon laquelle la campagne enferme dans le mépris et le retard, tandis que la ville est l'idéal, le lieu où peuvent s'ouvrir les possibilités économiques, sociales et de prestige” (cf. Albó et al., 1981 : 73).

(62) En 1989, 73,5\% du total de la Population Économiquement Active (PEA) de la ville de El Alto relevait du secteur informel de l'économie ( $c f$. Antezana Villegas, 1993 : 57 ).

(63) Voir entre autres pour ce concept : Durkheim, $1960 ; 1967$. 
familiale dont l'unité constitue pourtant une constante du discours du Compadre. Notre enquête montre aussi que ce sont les femmes de pollera séparées de leur époux qui sont les plus enclines à voter pour CONDEPA ( $c f$. Alenda, 2001a, à paraître).

3. Dans ce contexte de multiplication des stratégies de survie, la perception de l'inégalité des chances de réussite (qui est, il va de soi, fonction des aspirations des acteurs) tend à s'accentuer pour les femmes de pollera les mieux intégrées à l'univers urbain. Cette lecture de la réalité sociale s'inscrit dans une tradition sociologique (Tocqueville, 1981 : tomes I et II ; Inglehart, 1977) qui prétend montrer que les acteurs les plus contestataires ne sont pas forcément les plus pauvres (64). Appliqué à notre cas, ce paradoxe apparent peut être compris de la façon suivante : la frustration relative (65) des femmes de pollera tend à augmenter dès lors qu'elles occupent une position sociale “ privilégiée " au sein de leur propre in-group (Hyman, 1942) — c'est-à-dire lorsqu'elles sont nées à El Alto, qu'elles ont un niveau d'étude secondaire et un revenu supérieur au salaire moyen - , parce que ce statut particulier va de pair avec une perception intensifiée de l'inégalité face à la réussite (relativement aux hommes et aux femmes de vestido) étant donné le stigmate inhérent à la condition de femme de pollera. En ce sens, la frustration relative ne procède pas tant d'une inégalité objective des chances que du rapport plus ou moins enchanté à une position sociale. La visibilité des inégalités découle ainsi d'une plus grande confrontation à celles-ci, elle-même conditionnée par la concurrence. Si la condition sociale est plus fréquemment remise en question dans le cas où les actrices occupent une place privilégiée au sein de leur propre groupe, cela n'est pas dû à leur plus grande capacité (66) ou légitimité à avoir une opinion politique, mais au fait qu'une meilleure connaissance des règles du jeu urbain rend manifestes les limites de l'ascension. Plus la frustration des cholas est forte, plus il est probable qu'elles protestent contre un ordre social qui leur est défavorable en votant pour CONDEPA. C'est, du reste, ce qui contribue à expliquer que leur vote pour CONDEPA soit approximativement deux fois plus important (Alenda, 2001a; 2001b — à paraître —) que le vote des hommes et des femmes de vestido.

De même qu'il transforme l'identité populaire en identité positive, valorisée, le Compadre, secondé par Remedios Loza " relève les femmes de pollera" (pour parler comme les militantes), encourage leur dépassement et l'oubli de leur stigmate. La manière dont une militante perçoit la Comadre est, à ce propos, tout à fait éloquente :

“ Ce que j'apprécie par-dessus tout en elle [Remedios Loza] c'est sa façon de se dépasser, nous savons qu'elle est une personne aymara, elle a été chola, et ce que j'admire c'est sa façon d'évoluer [superarse], au fond le vêtement lui importe peu [no le interesa], le fait qu'elle soit de pollera 1972).

(64) Ce que signalait Lavaud dans une étude sur le quartier pacénien de Achachicala (Lavaud,

(65) Pour la notion de "frustration relative ", voir entre autres auteurs Merton, 1997. Pour le détail de notre argumentation, nous renvoyons à Alenda, 2001a (à paraître).

(66) Au sens où l'entend Bourdieu pour lequel le “ droit statutaire " sur la politique est supposé croître à mesure qu' augmente le niveau d'études (1979 :478). Bourdieu donne à ce concept deux sens : d'une part, une capacité proprement politique à répondre à la question politique posée ; d'autre part, une capacité socialement reconnue à donner un avis politique (1979 : 466). 
lui importe peu [no le interesa], ce que j'admire le plus en elle, c'est son évolution" (67).

En s'élevant dans l'échelle sociale, Remedios a également réussi à renverser la négativité de son identité de chola : elle a, pourrait-on dire en paraphrasant cet extrait d'entretien, " cessé d'être chola".

\section{CONCLUSION}

Il s'est agi dans cet article d'examiner la pertinence pour l'étude des néopopulismes de postulats communs aux théories classiques du populisme qui continuent d'orienter de façon plus ou moins revendiquée les travaux actuels sur ce type de mobilisation collective : la manipulation des masses par un leader charismatique, leur absence de conscience de classe ou leur attachement à des valeurs traditionnelles, pour ne pas dire prémodernes. Nous avons opté pour une méthodologie sociologique à la fois descriptive et compréhensive d'une forme d'action collective sur laquelle il reste énormément à faire.

D'une part, l'étude du condépisme ne valide pas l'hypothèse d'une mobilisation populaire équivalant à une résistance au changement (elle ne valide pas non plus l'inverse, à savoir l'uniformisation des masses apathiques sous l'effet des médias). Nous avons vu au contraire que ceux qui tendaient le plus à voter pour CONDEPA étaient ceux qui, parmi les plus discriminés, étaient aussi les mieux intégrés à l'univers urbain où ils mettent en œuvre des tactiques individuelles d'ascension sociale.

D'autre part, avant de fonder ou de réfuter l' argument de l'absence de conscience de classe des couches populaires, peut-être faudrait-il s'interroger sur la pertinence de la question. Selon Favre, à partir des années 80 :

“les sociétés latino-américaines affectent une configuration nouvelle. Le clivage fondamental qui les traverse ne passe plus entre les classes. Il oppose désormais un secteur qui demeure stratifié, organisé et associé directement à la production, à un secteur périphérique, inorganique et massifié que l'appareil économique maintient en marge du processus productif (1996 : 109)".

Dans ce contexte, parmi les individus économiquement inutiles (acteurs d'une économie informelle) en quête d'intégration (68), s'opère une érosion des identités collectives bien que la Bolivie ne présente pas, du moins pendant les dix ans de condépisme, le degré d'anomie décrit par Favre pour l'ensemble des pays latinoaméricains.

Ce contexte est dès lors propice au développement de mécanismes d'identification (69) (à Radio Télévision Populaire, Conciencia de Patria, Carlos Palenque ou Remedios

(67) Entretien avec Felipa, El Alto, octobre 1995.

(68) Une caractéristique que les populismes des années 30-50 partagent avec les néopopulismes des années 89-90 (seule change la forme de l'État entre 1950 et 1980).

(69) Dont la consolidation dépend de divers facteurs combinés tant conjoncturels que structurels : médiatiques, politiques et économiques. Le lien avec le Compadre se trouve renforcé par 
Loza), lesquels, en renversant la discrimination, renversent du même coup le fatalisme populaire (70) (ce que démontrel'un des slogans scandé par les condépistes : "Compadre Palenque, tu es l'espoir"). Ces mécanismes agissent alors comme des principes socialisateurs, organisant une réalité incertaine et favorisent l'émergence d'identités collectives (le peuple, les femmes de pollera) modelées par une idéologie du progrès et du droit à avoir des droits. En ce sens, le populisme peut être vu comme un processus d'affirmation subjective (d'un sujet populaire) qui incarne le changement à défaut de le susciter (71).

Ce changement, inscrit dans un contexte qui ne cesse pas d'être défavorable à l'émergence d'une conscience de classe, s'exprime à travers l'autonomisation des acteurs qui n'exclut pourtant pas le verticalisme et l' " hyperpersonnalisation "(Taguieff, 1996) du mouvement palenquiste au sein duquel tout semble confluer en direction du leader en une sorte d'idéal-type du populisme (72). Même si le discours de Remedios possède à l'évidence des effets amplificateurs sur l'émancipation de la femme de pollera, il entraîne en même temps, comme nous l'avons dit, des effets pervers. Dans le même ordre d'idées, certaines déclarations des Alténiens que nous avions interrogés sur l'avenir de CONDEPA sans Palenque (73) laissaient entrevoir une autonomisation du peuple auquel il ne restait plus qu'à suivre la voie " tracée par le Compadre ", " parce qu'il a tout laissé en place et il ne reste plus qu'à suivre " [Porque él dejó hecho todo y no es más que seguir], parce que " Carlos Palenque vit en chaque condépiste " ou encore parce que " Nous pouvons tous être des Palenques (74)"... Il semblerait que ce degré d'autonomie de l'acteur entendue comme " la capacité de se reconnaître lui-même dans ses œuvres et dans ses projets, de coopérer au pilotage de son propre développement, de découvrir en celui-ci une exigence de sa propre réalisation" (75) rende quelque peu réductrice la perception du social en termes de reproduction des inégalités.

l'émotion succédant à la fermeture de RTP dont émerge un parti politique, par l'effet sur l'imaginaire des cholas de l'ascension sociale médiatisée d'une femme de leur condition, ou encore par le contexte compétitif de l'une des villes les plus pauvres de Bolivie : El Alto.

(70) Observé par Lavaud, 1972.

(71) Cf. Touraine cité par Moscoso: “(...) dans celui-ci [le populisme], les éléments d'identification sont plus importants que les éléments de réalisation " (1990 : 194).

(72) Cette même "pureté" du lien avec le leader caractérise l'APRA péruvien selon Mariátegui (cf. "El populismo y el APRA", 1994).

(73) La question était la suivante : Pourquoi (oui ou non) pensez-vous que CONDEPA continuera après la mort de Carlos Palenque?

(74) Ces réponses ont été recueillies lors de notre enquête par sondage, El Alto, mai-juin 1997.

(75) Selon la lecture de la notion d'autonomie de Durkheim par Boudon \& Bourricaud, $1982: 122$. 


\section{Références citées}

ALBO, J., GREAVES, T. \& SANDOVAL, G., 1981 - CHUKIYAWU, La cara aymara de La Paz, I. El paso a la ciudad, 150p.; La Paz : CIPCA.

ALENDA, S., 2001a-Métissage culturel et politique en Bolivie. Émergence et audience du parti Conscience de la Patrie (CONDEPA). Thèse de doctorat, Université de Lille 1, à paraître.

ALENDA, S., 2001b - "La representación política de las cholas en Bolivia: la influencia de Conciencia de Patria (CONDEPA) en la construcción de nuevas identidades urbanas". In: Actes du 50 Congrès International des Américanistes, Varsovie, 9-14 juillet 2000, à paraître.

ÁLVAREZ JUNCO, J., 1994 - El populismo como problema. In : El populismo en España y América (J. Alvarez Junco \& R. González Leandri, comp.) : 11-38 ; Madrid : Editorial Catriel.

ANTEZANA VILLEGAS, M., 1993 - El Alto desde El Alto II, 356p. ; La Paz : UNITAS.

ARCHONDO, R., 1991 - Compadres al micrófono, 249p. ; La Paz : HISBOL.

ARCHONDO, R., 1997 - Compadres al micrófono. In : Documentos Hoy: El Compadre, su vida, sus pasiones y su muerte : 2-3 ; La Paz, 16 de marzo de 1997.

ARENDT, H., 1958 - The origins of Totalitarianism, 520p. ; London : Allen and Unwin.

BAPTISTA GUMUCIO, M., 1996 - Breve Historia contemporánea de Bolivia, 391p. ; México : Fondo de Cultura Económica.

BONNET, N., 2000 - La Bolivie, terrain d'expérimentation d'une approche globale du développement. Le Monde, 9 septembre 2000.

BOUDON, R. \& BOURRICAUD, F., 1982 - Dictionnaire critique de la sociologie, 714p. ; Paris : PUF.

BOURDIEU, P., 1979 - La distinction, 670p. ; Paris : Les Éditions de minuit.

CAMPBELL, A., CONVERSE, P. E., MILLER, W. \& STOKES, D., 1960 - The American Voter, 558p. ; Survey Research Center, University of Michigan.

CARDOSO, F. H. \& FALETTO, E., 1969 - Dependencia y Desarrollo en América Latina. Ensayo de interpretación sociológica, 167p.; México : ED. Siglo XXI.

DEMÉLAS, M.-D., 1992 - L'Invention Politique, 620p. ; Paris : Éditions Recherche sur les Civilisations.

DI TELLA, T., GERMANI, G. \& GRACIARENA, J., 1965 - Argentina, Sociedad de Masas, 288p. ; Buenos Aires : Editorial Universitaria de Buenos Aires.

DOBRY, M., 1993 - Logiques de la fluidité politique. In: Action collective et mouvements sociaux (sous la direction de F. Chazel) : 177-182 ; Paris : PUF.

DURKHEIM, É., 1960 - Le Suicide, étude de sociologie, 461p. ; Paris : PUF.

DURKHEIM, É., 1967 - De la division du travail social, 416p. ; Paris : PUF.

FAVRE, H., 1976 - Pour un modèle alternatif de la société andine. In : INSERM : 73-86. Vol. 63, août.

FAVRE, H., 1996 - L'indigénisme, 128p. ; Paris : PUF.

FREUD, S., 1981 - Psychologie des foules et analyse du moi. In: Essais de psychanalyse : 117217 ; Paris : Payot.

FURET, F., 1978 - Penser la Révolution française, 316p. ; Paris : Gallimard.

GERMANI, G., 1962 - Política y Sociedad en una época de transición: de la sociedad tradicional a la sociedad de masas, 266p. ; Buenos Aires : Ed. Paidos.

GERMANI, G., 1978 - Authoritarianism, Fascism and National Populism, 292p. ; New Jersey : Transaction Books.

HYMAN, H., 1942 - The psychology of status. Archives of Psychology, nº 249. 
IANNI, O., 1975 - La formación del Estado populista en América latina, 179p. ; México : Ed. Era.

INGLEHART, R., 1977 - The Silent Revolution: changing values and political styles among Western publics, 482p. ; Princeton University Press.

KLAPPER, J. T., 1960 - The effects of Mass communication, 302p. ; Illinois : The Free Press of Glencoe.

LACLAU, E., 1977 - Politics and ideology in Marxist theory: capitalism, fascism, populism, 203p. ; London : NBL Atlantic Highlands: Humanities Press.

LAVAUD, J.-P., 1976 - Compérage, stratification sociale et rapports de pouvoir : une enquête à La Paz. Cahiers des Amériques Latines, 13-14 : 103-125 ; Paris : IHEAL.

LAVAUD, J.-P., 1972 - Organisation sociale et attitudes politiques dans un quartier marginal de La Paz. Une enquête par questionnaires dans le " barrio " d'Achachicala, Thèse pour le doctorat de $3^{\text {ème }}$ cycle, Université René Descartes, Paris V, U.E.R. de Sociologie.

MACLUHAN, M., 1968 - Pour comprendre les médias, les prolongements technologiques de l'homme, 390p. ; Paris : Éds. du Seuil.

MANN, P., 1993 - Crise et attestation charismatique. In : Action collective etmouvements sociaux (sous la direction de F. Chazel) : 193-204 ; Paris : PUF.

MANSILLA, H. C. F., 1995 - La influencia de la empresa privada sobre las políticas públicas en Bolivia. In : Opiniones y Análisis, Comportamientos políticos en Bolivia : 155-176; La Paz : FUNDEMOS.

MERTON, R. K., 1997 - Éléments de théorie et de méthode sociologique, 384p. ; Paris : Armand Colin.

MESA, C., 1992 - Sobre el gonismo. In : Los nuevos actores políticos en Bolivia ; La Paz : ILDIS.

MESA, J. de, GISBERT, T. \& MESA, C. de, 1997 - Historia de Bolivia, 779p. ; La Paz : ed. Gisbert.

Ministerio de Desarrollo Humano, Unidad de Análisis de Políticas Sociales, Instituto Nacional de Estadística, Unidad de Políticas de Población, Unidad de Análisis de Políticas Económicas, diciembre 1993 - Mapa de pobreza, Una Guía para la Acción Social, 154p. ; La Paz.

Ministerio de planeamiento y coordinación, Instituto Nacional de Estadística, noviembre 1992 Encuesta Integrada de Hogares, 305p. ; La Paz.

MOSCOSO PEREA, C., 1990 - El populismo en América latina, 297p. ; Madrid : Centro de Estudios Constitucionales.

OPINIONES Y ANÁLISIS, 1998 - Datos estadísticos generales 1979-1997, Tomo I, 312p. ; La Paz : FUNDEMOs.

PALENQUE, C., 1995 - Discursos 94', 64p. ; La Paz : Ediciones P.P.

SAN MARTÍN, H., 1991 - El palenquismo, 188p. ; La Paz : Los Amigos del Libro.

SARAVIA, J. \& SANDOVAL, G., 1991 - Jach'a Uru: ¿la esperanza de un pueblo?, 239p. ; La Paz : ILDIS-CEP.

SCHRAMM, W. L., 1964 - Mass Media and national development. The role of information in the developing countries, 333p. ; Stanford University Press.

VEGA-CENTENO B., I., 1991 - Aprismo Popular, Cultura, Religión y Política, 599p. ; Lima : CISEPA/TAREA.

TAGUIEFF, P.-A., 1996 - Le populisme. In : Encyclopaedia Universalis : 118-125.

TOCQUEVILLE, A. de, 1981a - De la Démocratie en Amérique, Tome I, 569p. ; Paris : GarnierFlammarion.

TOCQUEVILLE, A. de, 1981b - De la Démocratie en Amérique, Tome II, 414p. ; Paris : GarnierFlammarion. 